\title{
Finanzierung von Öko-Innovation
}

\author{
Gottfried Lamers
}

Online veröffentlicht: 21.8.2008

(C) Die Autoren 2008

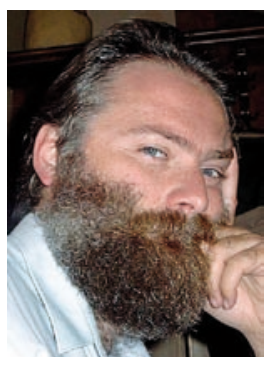

die Innovationspolitik beschränkt sich auf die Bereitstellung von Plattformen, auf denen sich Forscher und Marktteilnehmer begegnen. Plakativ gesprochen mischt sich der Staat bzw. die Politik nur sehr peripher in die Innovation z. B. bei Zahnbürsten ein. Lediglich die Sicherstellung und Weiterentwicklung von Standards und Normen wird in diesem Sektor vom Staat betrieben. Diese wiederum kommen einem Bedürfnis der Wirtschaft nach einem Mindestmaß an Regulierung nach, um allgemein kompatible Komponenten zu definieren. Manchmal dient diese Normung der Wirtschaft auch dazu ,lästige“ neue Mitbewerber vom Markt fernzuhalten. Eine Strategie, die meist nur zu Verzögerung, nicht jedoch zur Verhinderung interessanter Innovationen führt.

Im Bereich der „Öko-Innovation“ spielen jedoch der Staat und damit auch die Politik eine viel stärkere Rolle und sie beeinflussen den Markt wesentlich stärker. Teilweise ist dieser Markt sogar erst durch die Umweltpolitik geschaffen und entwickelt worden.

\section{Was ist „Öko-Innovation“" und warum funktioniert sie anders als herkömmliche Innovation?}

Innovation entsteht an der Schnittstelle zwischen Forschung und Markt. Damit ist bereits die Hauptschwierigkeit der Innovationspolitik beschrieben. Was braucht der Markt und welche Forschungsergebnisse sind tatsächlich verwendbar? Üblicherweise übernimmt der Markt dabei die Auswahl und

Gottfried Lamers $(\bowtie)$

Bundesministerium für Land und Forstwirtschaft, Umwelt und Wasserwirtschaft, Abteilung II/3 Nachhaltige Entwicklung und Umweltförderpolitik,

Stubenbastei 5, 1010 Wien, Österreich

E-Mail: gottfried.lamers@bmlfuw.gv.at

\section{Die politische Dimension der Öko-Innovation}

Konkret hängt der Markt auf zwei Arten von der Umweltpolitik ab.

- Einerseits schafft die Politik den Markt erst durch neue Umweltgesetze und Normen. Ohne entsprechende Verordnungen und technische Richtlinien würde kaum ein Betrieb freiwillig Abgasreinigungsmaßnahmen setzen. Erst die kontinuierliche Verschärfung von Normen und Grenzwerten weckt den Bedarf nach Anlagen und damit auch nach Forschung und Innovation bezüglich besserer und kosteneffizienterer Erfüllung dieser Normen. Als Konsequenz daraus haben daher auch jene Länder, die Normen früher einführen oder aktiv die Weiterentwick- 
lung der Grenzwerte betreiben, im Wettbewerb der Umweltindustrie führende Rollen.

- Andererseits tritt der Staat im Bereich der Umwelt auch stark als Marktteilnehmer auf. Primär Infrastrukturmaßnahmen im Wasser-, Abwasser- und Abfallbereich wer-

Ein kleines Beispiel aus Österreich:

2002 wurde in einer vorausschauenden Umweltpolitik und nach langen politischen Verhandlungen die Verwendung von fluorierten Chemikalien in Feuerlöschanlagen verboten (so wie in der Schweiz und Dänemark). Die Wirtschaft begann langsam (aufgrund der Auslauffristen) auf HFKW-23 freie Löschmittel umzusteigen. Lediglich ein Produzent dieser Löschmittel wollte das Ergebnis auch nach Jahren nicht akzeptieren. Er fand einen Formfehler in der Verordnung und nach 5 Jahren wurde er vom Verfassungsgerichtshof dahingehend bestätigt, dass die Verordnung geändert werden müsse. An sich ist das eine Formalität, jedoch muss auch die Berichtigung der Novelle einen politischen Prozess durchlaufen. Dabei stellte sich jedoch plötzlich heraus, dass der Produzent gute Lobbyarbeit betrieben hatte und ein Ministerium plötzlich der gleichen (lediglich um den Formfehler bereinigten) Verordnung nicht mehr zustimmte. Die Folge ist, dass jetzt diese Substanzen wieder zugelassen sind. Man kann sich ausrechnen, was das für jene Firmen bedeutet, die bereits in die teurere Technologie investiert haben. Die „early birds“ werden für ihre frühzeitige Entscheidung abgestraft, jene, die sich Zeit gelassen haben, können jetzt wieder auf billigere Löschmittel zurückgreifen. Besonders interessant ist dieser Modellfall jedoch für die Technologieanbieter. Jene Firmen, die - durch die gültige Verordnung ermutigt - Technologieentwicklung in neuen, HFKW freien Techniken betrieben haben, stehen jetzt vor dem Aus. Und mit ihnen alle Investoren und Banken. (Um die Dimension richtig zu stellen: Es handelt sich um einen kleinen überschaubaren Bereich und niemand ging tatsächlich bankrott, jedoch veranschaulicht das Beispiel sehr gut die Wirkungsmechanismen politischer Entscheidungen.)

Eine ähnliche Unsicherheit erzeugt die permanente Diskussion über Atomkraft in Deutschland. Einmal gibt es langfristige Ausbaupläne, dann einen „unwiderruflichen" Ausstieg aus der Atomkraft und dann wieder einen möglichen Neueinstieg. Investoren in Umwelttechnologie (wie Banken, Fonds etc.) sind gar nicht so sehr daran interessiert, was tatsächlich gebaut wird, sie wollen nur die Gewissheit, dass eine einmal getroffene Entscheidung nicht nach Wahlen wieder geändert wird. den vom Staat (in den meisten Fällen den Kommunen) selbst gesetzt und finanziert. Würde die öffentliche Hand als Investor wegfallen, würde auch damit der gesamte Markt und mit ihm die produzierende Industrie zusammenbrechen.

Diese starke Rolle des Staates und damit auch der jeweiligen Umweltpolitik ist jedoch auch die besondere Schwäche des Sektors und das größte Hemmnis der Öko-Innovation.

Im ersten Fall ist die Forschung und Wirtschaft abhängig von den Launen der Umweltpolitik und langfristige Geschäftsstrategien können durch simple Neuwahlen oder teilweise sogar Lobbygruppen über den Haufen geworfen werden (siehe Beispiel im linken Kasten).

Wenn der Staat - und über dessen Mittelallokation die Kommunen - als Hauptkunde auftreten, ist die Situation für Technologieentwickler und Investoren kaum besser. Jeder Konjunktureinbruch führt zu sofortiger Kürzung von staatlichen Infrastrukturprogrammen und damit zu einer ständigen Unsicherheit. Weiters führt die Struktur der Abnehmer (öffentliche Hand) oft zu übervorsichtigen Entscheidungen. Die Angst, wegen einer technischen Fehlentscheidung durch den Rechnungshof oder - schlimmer - die Wähler abgestraft zu werden, bewegt kommunale Investoren, bewährte, erprobte und damit durchschnittliche Technologien zu wählen. Der Abwassersektor ist dafür ein gutes Beispiel.

Ein kleines Beispiel aus Österreich:

Die Verlegung von Abwasserrohren hat zwei große Kostenfaktoren. Einerseits wird das Erdreich für die sog. „Künette“ ausgehoben und auf eine Deponie verfrachtet, andererseits frisches Füllmaterial aus Sandgruben und Steinbrüchen herbeigeschafft. Der Hauptgrund für den Einsatz des neuen Materials liegt in der kontrollierbaren Mischung der Füllung und somit der optimierten Verdichtungsmöglichkeit. Der Einsatz des Ursprungsmaterials könnte zu späteren Setzungen und Fahrbahnschäden führen. Über mehrere Forschungsprojekte (auch auf europäischer Ebene) wurde nachgewiesen, dass es einfache Analysemethoden gibt, das Aushubmaterial $\mathrm{zu}$ bestimmen und die Verdichtung darauf abzustimmen. Trotzdem wird diese viel billigere Methode kaum verwendet. Das „Restrisiko“ für die Kommune ist offenbar zu hoch. Die Technologieentwickler haben nach 10 Jahren Demonstration vermutlich auch schon aufgegeben. Investoren waren nicht zu finden.

Wenn gar kein Geld für Investitionen zur Verfügung steht, kann natürlich auch keine Technologieentwicklung erfolgen. 
Ein Beispiel aus Mazedonien:

Ein japanischer Technologieentwickler bietet dem Umweltministerium an, kostengünstige low tech Technologien zur Klärschlammtrocknung mit mazedonischen Firmen zu entwickeln. Diese gute Idee scheitert lediglich an der Tatsache, dass es in ganz Mazedonien keine einzige funktionierende Kläranlage gibt und demnach Klärschlamm noch für einige Jahrzehnte kein besonderes Problem bereiten wird.

Banken und andere Investoren bedienen sich allgemein üblicher Methoden der Marktabschätzung, bevor sie Geld vergeben. Marktanalysen und ähnliches sind für größere Investitionen im Technologiebereich Standard. Neue Computerspiele werden auf Markttauglichkeit getestet, lediglich bei neuen features im Telekombereich erspart man sich die Prüfung, da dort sowieso jeder neue Schnickschnack gekauft wird.

Im Bereich der Öko-Innovation stoßen jedoch die bekannten Analysemethoden an ihre Grenzen. Die technische Tauglichkeit einer Öko-Innovation reicht eben noch lange nicht aus, um den kommerziellen Erfolg abzuschätzen. Die schwierige Abschätzung politischer Risiken ist den Investoren offenbar durchaus bewusst und resultiert daher in äußerster Vorsicht. Öko-Innovationen müssen - um überhaupt an Kapital zu kommen - wesentlich höhere Gewinnaussichten bieten als normale Innovationen. Manche Banken verzichten daher ganz auf das Geschäft und überlassen es „Heuschrecken“, die natürlich für ihr Risiko entsprechende Gewinnaussichten erwarten. Eine Beteiligung eines venture capital funds ist erfolgreich, wenn der Investor nach 3-5 Jahren mit $80 \%$ Gewinn aussteigen kann. Eine Wertsteigerung des Unternehmens um „nur“ $30 \%$ wird schon als Misserfolg betrachtet. Fairerweise muss man dazusagen, dass ca. 60-70\% der Beteiligungen gar keinen Gewinn abwerfen und so eben die erfolgreichen Innovationen diesen Ausfall kompensieren müssen.

Privates Risikokapital ist daher prinzipiell nur verfügbar, wenn die Technologie auch auf den ersten Blick sehr hohe Renditemöglichkeiten verspricht. Durchschnittliche oder gar nur moderate Renditen haben keine Attraktivität und damit auch kaum Zugang zu diesem Kapital. Das ist auch der Beweggrund der Europäischen Kommission, in diesem Bereich staatliche bzw. EU Förderungen anzubieten. Durch die Beteiligung des European Investment Funds an venture capital funds sinkt das Risiko der privaten Fondsbetreiber und es werden auch Projekte mit etwas geringerer Performanceoption finanziert. Das competitiveness and innovation programme (CIP) bietet derartige Förderungen für Fondsbetreiber mit dem Fokus auf Umwelttechnologien an.
Bei nüchterner Analyse des Programms ist jedoch ablesbar, dass die Attraktivität für derartige Finanzierungsformen auch wieder von umweltpolitisch stabilen Mitgliedstaaten genutzt wird. Offenbar reagiert auch das private Risikokapital (trotz durch Förderung abgemildertem Risiko) skeptisch auf umweltpolitische Instabilität oder Umbrüche.

\section{Schnittstellenproblematik}

Neben den oben skizzierten politischen Hürden für die Finanzierung von Öko-Innovationen gibt es auch strukturelle und administrative Probleme. Innovation liegt immer an Schnittstellen, die Öko-Innovation hat hier aber auch noch einige zusätzliche gegenüber der herkömmlichen Innovation aufzuweisen.

Es gibt Schnittstellen zwischen:

- Technologieentwicklern und -anwendern

- Forschungs- und Umweltministerien

- Forschungs- und Umweltförderungen.

\section{Produzent oder Konsument}

Die Schnittstellen zwischen Technologieentwicklern und den Anwendern haben alle Innovationen gemeinsam. Technologien werden in Forschungseinrichtungen entwickelt und von interessierten Produzenten aufgenommen und zur Marktreife weitergetrieben. In diesem Übergang von Wissenschaft zu Produzent geht bereits ein Großteil an innovativen Ideen verloren (aufgrund geringer Marktchancen). In dieser Phase haben Umwelttechnologien noch mit den gleichen Problemen wie alle anderen Innovationen zu kämpfen.

An der Schnittstelle der Produzenten mit den Kunden zeigt sich aber wieder die bereits beschriebene schwierige Struktur des Anwenderkreises. Die Entwickler der Technologien versuchen dann über Öko-Innovationen (also Demonstrations- und Pilotprojekte) die neuen Produkte in den Markt einzuführen. Dabei brauchen sie jedoch interessierte Partner, die bereit sind, diese neuen Technologien auch anzuwenden. Das heißt jedoch, dass auch der Anwender bereit sein muss, ein höheres technologisches Risiko zu tragen. Insbesondere Anwender der öffentlichen Hand sind äußerst risikoavers und kaum bereit, technische Risiken einzugehen. Es hat sich daher bewährt, Plattformen von Forschung, Produzent und Anwender einzurichten, in denen sich Partner bereits von Beginn an zusammenschließen, um bestehende Probleme zu lösen. Entscheidend zur Überwindung dieser Schnittstelle sind Partnerbörsen bzw. Technologiemessen etc. Der Staat kann in diesem Spannungsfeld auf verschiedene Weise unterstützend eingreifen. Über Technologieplattformen oder die Unterstützung von Messen kann 
einerseits diese Kontaktanbahnung forciert werden, andererseits damit auch das Interesse der Politik an den Lösungen dokumentiert werden. Das Ausmaß dieser politischen Unterstützung dient oft als Gradmesser der Chancen einer Technologie und ist daher für das Engagement von Unternehmen entscheidend. Oft wird man als Ministeriumsmitarbeiter noch nach Jahren (und diversen personellen Änderungen bei den Ministern) auf Interviews und Aussagen der (längst verflossenen) PolitikerInnen angesprochen, die als Grundlage für Unternehmensentscheidungen herangezogen wurden.

\section{Administrative Kompetenzkonflikte}

Die Schnittstelle der Ministerien ist ein Problem, das nur die Öko-Innovation kennt. Während sich kein Ministerium dezidiert um die Innovationen im (um beim vorherigen Beispiel zu bleiben) „Zahnbürstensektor“ kümmert, sind es im Bereich der Umwelttechnologien mindesten zwei. Wenn es sich um Innovationen im Verkehrsbereich handelt, kommt meistens auch noch das Verkehrsministerium dazu. Technologieentwickler sind daher manchmal mit divergierenden Aussagen der politischen Ebene konfrontiert. So sehr ein positiver Kompetenzkonflikt auch als politisches Signal gewertet werden kann (alle Ministerien kümmern sich rührend um die Umwelttechnologie) so mühsam ist es für die Produzenten/Anwender die letztlich wirklich zuständigen Referenten und Dienststellen zu finden. Ohne klare schriftliche Kompetenzteilung zwischen den Ressorts führt nämlich der positive Kompetenzkonflikt auf oberster Hierarchieebene dann zu negativen Konflikten auf Sachbearbeiterebene. Öko-Innovationsanbieter werden daher - trotz bekundeter politischer Unterstützung - letztendlich im Kreis geschickt, um die tatsächlichen Ansprechpartner ausfindig zu machen.

Diese Situation lässt sich nur dadurch lösen, dass neben den politischen Sonntagsreden ganz konkrete Kooperationsvereinbarungen auf unterer Hierarchieebene getroffen werden. Damit kann Informationsfluss, Austausch und Abstimmung einzelner Programme sichergestellt und auch die einzelnen MitarbeiterInnen der Administrationen können in die generelle Öko-Innovationspolitik eingebunden werden.

\section{Subventionsdschungel}

Die dritte Schnittstelle ist jene zwischen den involvierten Förderungsinstrumenten. Im Gegensatz zur beschriebenen Kooperation im politischen Bereich, bedarf das Förderungswesen einer klaren Abgrenzung. Dies auch deshalb, da die Förderungen nicht nur nationalen, sondern auch europarechtlichen Regularien unterliegen. Insbesondere die klare Trennung zwischen dem anwenderorientierten Umweltförderungssystem und dem entwicklerzentrierten Forschungsrahmen macht die Finanzierung von Demonstrationsprojekten besonders komplex. Forschungsförderungen (nach dem gemeinschaftsrechtlichen Forschungsförderungsrecht) richten sich an die Produzenten und Forscher. Mit diesen Subventionen können zwar Entwicklungskosten, Forschung (also Arbeitszeit) sowie Verifizierung mit hohen Förderungssätzen gefördert werden, jedoch keine Infrastruktur (also Baukosten). Im Umweltrahmen hingegen können vorrangig Investitionskosten gefördert werden und der Fokus liegt auf den Anwendern. Die Förderung von Demonstrationsprojekten mit zusätzlicher Forschung gestaltet sich daher prinzipiell als komplex, da verschiedene Systeme vereint werden müssen. Auch in diesem Fall bedarf es einer intensiven Kooperation und gegenseitigen Abstimmung der beteiligten Ministerien und Förderungsstellen.

\section{Lösungsansätze}

Öko-Innovation hat in zahlreichen Bereichen schlechtere Bedingungen als normale Innovationen. Allerdings stehen für Umwelttechnologien auch viel mehr finanzielle Mittel zur Verfügung. Das Problem der Öko-Innovation ist daher nicht vordringlich ein finanzielles.

\section{Risikominimierung}

Wie am Anfang diskutiert, sind ein Hauptgrund der Unsicherheit die hohen politischen Risiken der Einführung neuer Umwelttechnologien. Umweltpolitische Zielsetzungen sollten daher in mittel- bis langfristigen Perspektiven zusammengefasst und breit politisch akkordiert werden. Je öffentlicher die Partizipation ausgelegt ist, desto schwieriger sind kurzfristige tagespolitische Änderungen möglich und desto stabiler ist das Gesamtsystem. Dadurch erst kann das Vertrauen von Technologieentwicklern, Investoren und Banken gewonnen werden.

\section{Finanzielle Strategien}

In jenen Bereichen, in denen die öffentliche Hand der Hauptauftraggeber ist, sollten die politischen Zielsetzungen auch mit ganz klaren finanziellen Strategien hinterlegt werden. Es ist besser zu signalisieren, dass nur wenig Mittel investiert werden können (die dafür aber sicher), als durch übertrieben optimistische Szenarien Unternehmen in Investitionen zu locken, die durch die ausbleibenden staatlichen Investitionen abgeschrieben werden müssen. International agierende Firmen verlassen in diesem Fall das Land und sind nur schwer zum Zurückkommen zu überreden. 


\section{Risikokapital}

Risikokapital aus venture funds oder Garantieinstrumenten können die Probleme in ausgewählten Bereichen mildern. Für die breite Umsetzung von Öko-Innovationen müssen die Banken gewonnen werden. Risikominimierung ist dabei ein Aspekt, die Schulung von Banken zur korrekten Risikobewertung ist eine andere Möglichkeit den Kapitalengpass zu überwinden. Eine enge Kooperation zwischen staatlicher Administration und Banken, allenfalls unter Auflage eigener Schulungsprogramme, ist dafür erforderlich.

\section{Institutionalisierte Begegnung}

Technologieplattformen, Technologiezentren etc. sind Einrichtungen zur Kooperation von Technologieentwicklern und -anwendern. Derartige Institutionen sind - vor allem in Verbindung mit innovationsorientierter Regionalpolitik ein wichtiger Baustein zur Gründung von Netzwerken und Clustern geworden. Je stärker die thematische Fokussierung der staatlich unterstützen Institutionen ist, desto größer waren die Erfolgschancen.

\section{Interministerielle Kooperation}

Regierungsziele zur Forcierung von Öko-Innovationen (etwa zur Erreichung der „Lissabon Ziele“) dürfen nicht auf
Ebene gemeinsamer Ministererklärungen stecken bleiben. Für die Umsetzer von Öko-Innovationen sind klar durchschaubare Kompetenzen auf unterster Ebene wichtiger als politische Ankündigungen. Besondere Bedeutung hat dabei eine institutionalisierte Kooperation.

\section{Förderungspakete}

Nicht neue Förderungsinstrumente sind gefragt, sondern die Koordination, Abstimmung und Abgrenzung bestehender Instrumente. Insbesondere für Projekte, die sich nicht in übliche Förderungsschemata pressen lassen sind flexible Förderungsangebote zu schnüren. Die nationalen Förderungsinstrumente brauchen daher auch entsprechende Flexibilität, um ohne ständigen Rechtfertigungsdruck gegenüber den politisch Verantwortlichen oder der Europäischen Kommission derartige Spezialförderungspakete schnüren zu können.

Um es noch einmal plakativ zusammenzufassen: ÖkoInnovation braucht keine politischen Gipfelstürmer oder „den großen Wurf“. Öko-Innovation braucht die Mühen der Ebene, viel Stabilität, Koordination und Kooperation auf allen Ebenen.

Open Access Dieser Artikel wird zu den Bedingungen der „Creative Commons Attribution Noncommercial License“ zur Verfügung gestellt. Damit ist eine nichtkommerzielle Nutzung, Verbreitung und Vervielfältigung erlaubt, sofern die Autoren des Artikels und die genaue Quelle angegeben sind. 